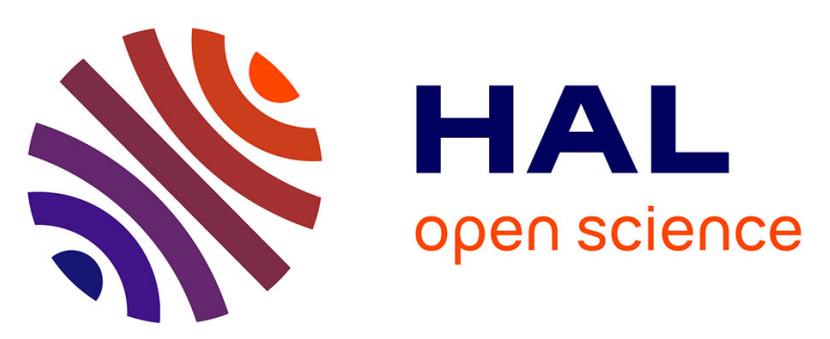

\title{
Analysis of a new variational model to restore point-like and curve-like singularities in imaging
}

Gilles Aubert, Laure Blanc-Féraud, Daniele Graziani

\section{To cite this version:}

Gilles Aubert, Laure Blanc-Féraud, Daniele Graziani. Analysis of a new variational model to restore point-like and curve-like singularities in imaging. Applied Mathematics and Optimization, 2013, 67, pp.73-96. inria-00522098v5

\section{HAL Id: inria-00522098 https://hal.inria.fr/inria-00522098v5}

Submitted on 14 Sep 2012

HAL is a multi-disciplinary open access archive for the deposit and dissemination of scientific research documents, whether they are published or not. The documents may come from teaching and research institutions in France or abroad, or from public or private research centers.
L'archive ouverte pluridisciplinaire HAL, est destinée au dépôt et à la diffusion de documents scientifiques de niveau recherche, publiés ou non, émanant des établissements d'enseignement et de recherche français ou étrangers, des laboratoires publics ou privés. 


\title{
Analysis of a new variational model to restore point-like and curve-like singularities in imaging ${ }^{1}$
}

\author{
GILLES AUBERT \\ LABORATOIRE J.A. DIEUDONNÉ \\ Université de Nice SOPHIA ANTIPOLIS, Parc Valrose 06108 Nice CEDEX 2, FRANCE \\ e.mail: gaubert@unice.fr \\ LAURE BLANC-FÉRAUD \\ MORPHEME CNRS/INRIA/UNSA SOPHIA ANTIPOLIS \\ Inria, 2004 route des Lucioles BP 9306902 SOPHIA ANTIPOLIS CEDEX, FRANCE \\ e.mail: Laure.Blanc-Feraud@inria.fr \\ DANIELE GRAZIANI ${ }^{2}$ \\ MORPHEME CNRS/INRIA/UNSA SOPHIA ANTIPOLIS \\ Inria, 2004 route des Lucioles BP 9306902 SOPHIA ANTIPOLIS CEDEX, FRANCE \\ e.mail: Daniele.Graziani@inria.fr \\ e.mail:daniele.graziani@gmail.com
}

\begin{abstract}
The paper is concerned with the analysis of a new variational model to restore point-like and curve-like singularities in biological images. To this aim we investigate the variational properties of a suitable energy which governs these pathologies. Finally in order to realize numerical experiments we minimize, in the discrete setting, a regularized version of this functional by fast descent gradient scheme.
\end{abstract}

AMS subject Classification: 65K10 49J45 49M20 49Q20

Keywords: biological images, non smooth convex optimization, $l^{1}$-minimization, Nesterov scheme, divergence-measure fields, $p$-capacity, relaxation.

\section{INTRODUCTION}

In biological image processing one might be interested in restoring an image, possibly corrupted by noise, characterized by an high intensity value on sets of low dimension, such as points (for example spot due to molecule) or curves in 2-D (for example filaments) and 3-D images and which decreases toward 0 in a neighborhood of the singularities. Then across these sets there is no jump of the gradient of the image. This phenomenon requires a new approach, which is different from the classical denoising

\footnotetext{
${ }^{1}$ The research of Daniele Graziani was supported until January 2010 by ANR under the research project "Detectfine" (Laboratory I3S, Université de Nice Sophia antipolis).

${ }^{2}$ Corresponding author.
} 
model based on the gradient operator. From a general point of view in order to restore the given data $u_{0}$, one would like to minimize an energy with an $L^{2}$-fidelity term and a proper regularization criterion F (assuming Gaussian noise or Gaussian approximation). To this end one has to solve the following minimum problem:

$$
\min _{u \in X} F(u)+\frac{\lambda}{2}\left\|u-u_{0}\right\|_{2}^{2}
$$

$\lambda>0$ is a positive weight, the functional space $X$ and the criterion $F$ must be chosen according to the singularities to be restored. In our case it means that $F$ must be given in term of a proper differential operator which is singular in a suitable sense on points and curves.

In this paper we are interested in function $u$, that locally behaves like the solution of the Dirichlet problem:

$$
\begin{cases}-\Delta u=\mu & x \in B \\ u=0 & x \in \partial B\end{cases}
$$

where $\mu$ is a Radon measure concentrated on points or open curves with boundary inside $\Omega$ (where $\Omega \subset \mathbb{R}^{N}$ is an open set) and finally $B$ is a neighborhood of the support of the measure $\mu$.

In fact our approach is inspired from the electrostatic and potential theory. For example we modelize a spot as a charged electrical particle. The resulting potential satisfies locally a PDE as (1.2) where $\mu$ is a Dirac mass concentrated on the spot. In the language of image processing the potential is called image intensity. Similar ideas can be developed for electrically charged lines or filaments.

We will show with two examples that the $L^{p}$-norm is not relevant for managing such singularities, while the variation of the Laplacian measure is on the contrary quite relevant (see Subsection 2.3 for examples). Despite the gradient is not sensitive to the singularities we want to preserve, we still need a regularization term depending on the gradient in order to be sure to remove as much as possible the noise. Indeed the Laplacian by itself does not guarantee strong regularization property because of cancellation effect due to its divergence form.

Hence, from a variational point of view, we have to find a functional space whose elements are able to produce Radon measures, whose support contains the singularities we would like to preserve. Then, in line with recent works for detecting point-like target problem (see $[5,14,15]$ ), we consider the divergence as an operator able of producing Radon measures concentrated on points and curves. We deal with the space $\Delta \mathcal{M}_{\text {loc }}^{p}(\Omega)$ of $W_{\text {loc }}^{1, p}$-functions whose gradient is an $L^{p}$-vector field with distributional divergence given by a Radon measure, where $1<p<\frac{N}{N-1}, \Omega \subset \mathbb{R}^{N}$ is the image domain and $N$ is the space dimension (see Section 2 below). The restriction on $p$ is due to the fact that when $p \geq \frac{N}{N-1}$ the distributional divergence $\operatorname{Div} \nabla u$ of $\nabla u$ cannot be a measure concentrated on points (see [5, 15]). Then to restore point-like and curve-like singularities in $2 \mathrm{D}$ or $3 \mathrm{D}$, we consider general criterion of type

$$
F\left(u, \Omega^{\prime}\right):= \begin{cases}\int_{\Omega^{\prime}} f(\Delta u) d x+\int_{\Omega^{\prime}} g(\nabla u) d x & \text { on } C^{2}(\Omega), \\ +\infty & \text { on } \Delta \mathcal{M}_{\mathrm{loc}}^{p}(\Omega) \backslash C^{2}(\Omega),\end{cases}
$$

where $\Omega^{\prime} \subset \subset \Omega$ is an open set; $f, g$ are convex functions with linear and $p$-growth from above respectively. 
Then, since in general functional (1.3) is not lower semicontinuous with respect to the $W_{\text {loc }}^{1, p}$-weak convergence, we deal with its lower semicontinuous envelope (or relaxed functional) on the space $\Delta \mathcal{M}_{\text {loc }}^{p}(\Omega)$.

So the first goal of the paper is to provide an integral representation result for the relaxed functional of functional (1.3), which guarantees that the regularization criterion we have chosen, matches with the singularities we aim to preserve. When $1<p<\frac{N}{N-1}$ we provide such a representation by using the notion of $p$-capacity which is a useful tool to distinguish sets of 0 -Lebesgue measure. In fact it turns out that, to extend functional (1.3) to the whole space $\Delta \mathcal{M}_{\mathrm{loc}}^{p}(\Omega)$, one has to measure the absolutely continuous part of the distributional divergence of $\nabla u$ and, at the same time, control its singular parts, encoded in two mutually singular measures $\mu^{a}$ concentrated on sets of positive $p$-capacity such as $2 \mathrm{D}$ curves or $3 \mathrm{D}$ surfaces, and $\mu^{0}$ concentrated on sets with $0 p$-capacity, such as points in $2 \mathrm{D}$ or curves in $3 \mathrm{D}$ (see for instance $[13,17,23]$ for general properties of $p$-capacity). Indeed by applying the $p$-capacitary decomposition, proven in [10], to the singular part, given by the Radon-Nykodim decompostion, of the measure $\operatorname{Div} \nabla u$, we have

$$
\operatorname{Div} \nabla u=\Delta u d x+\operatorname{Div}^{s} \nabla u=\Delta u d x+\mu^{a}+\mu^{0},
$$

(see subsection 2.2 below). Then we prove that according to previous decomposition, the lower semicontinuous envelope of $F$ is given by:

$$
\mathcal{F}\left(u, \Omega^{\prime}\right):=\int_{\Omega^{\prime}} f(\Delta u) d x+\int_{\Omega^{\prime}} g(\nabla u) d x+\int_{\Omega^{\prime}} f^{\infty}\left(\frac{d \mu^{a}}{d\left|\mu^{a}\right|}\right) d\left|\mu^{a}\right|+\int_{\Omega^{\prime}} f^{\infty}\left(\frac{d \mu^{0}}{d\left|\mu^{0}\right|}\right) d\left|\mu^{0}\right|,
$$

where $u \in \Delta \mathcal{M}_{\text {loc }}^{p}(\Omega), \Omega^{\prime} \subset \subset \Omega$ and $f^{\infty}$ is the recession function given by $\lim _{s \rightarrow+\infty} \frac{f(s t)}{s}$, with $t \in \mathbb{R}$. $\frac{d \mu^{a}}{d\left|\mu^{a}\right|}, \frac{d \mu^{0}}{d\left|\mu^{0}\right|}$ are the Radon-Nikodym derivatives of the measures $\mu^{a}$ and $\mu^{0}$ with respect to their total variation (see Theorem 3.1) and $\Omega^{\prime} \subset \subset \Omega$ open set.

In the second part of this work we focus on concrete applications in biological image processing in dimension 2. In practice we consider the following functional

$$
\mathcal{J}\left(u, \Omega^{\prime}\right):=\int_{\Omega^{\prime}}|\Delta u| d x+\frac{1}{p} \int_{\Omega^{\prime}}|\nabla u|^{p} d x+\left|\mu^{a}\right|\left(\Omega^{\prime}\right)+\left|\mu^{0}\right|\left(\Omega^{\prime}\right)+\frac{\lambda}{2} \int_{\Omega^{\prime}}\left|u-u_{0}\right|^{2} d x,
$$

which corresponds to the particular case $f=|\cdot|$ and $g=|\cdot|{ }_{p}^{p}$.

As a consequence of the relaxation result, we derive the existence of a unique minimizer of $\mathcal{J}$ on the space $\Delta \mathcal{M}_{\text {loc }}^{p}(\Omega)$ and that $\mathcal{J}$ is the lower semicontinuous envelope of

$$
J\left(u, \Omega^{\prime}\right):= \begin{cases}\int_{\Omega^{\prime}}|\Delta u| d x+\int_{\Omega^{\prime}}|\nabla u|^{p} d x+\frac{\lambda}{2} \int_{\Omega^{\prime}}\left|u-u_{0}\right|^{2} d x, & \text { on } C^{2}(\Omega), \\ +\infty & \text { on } \Delta \mathcal{M}_{\mathrm{loc}}^{p}(\Omega) \backslash C^{2}(\Omega),\end{cases}
$$

Then, by adapting some ideas of $[18,21,22]$ to our setting, we minimize the discrete version (see Subsection 4.1 for notation and definition in the discrete setting) of $J$ by a fast descent gradient algorithm.

Finally we test the proposed model to both synthetic and biological real images provided for us by "Institut Pasteur de Paris".

Let us conclude this introduction by pointing out that, of course, the idea of using second order differential operator is not new in image processing. One could mention, among the others, the recent 
work on second order generalized total variation of [4] or older based on Blake-Zisserman model (see for instance [6]). Nevertheless these models appear different from the one we propose here, since they focus on better preservation of jump discontinuities such as contour or corner, while we are interested in the restoration of different kind of singularities here.

The paper is organized as follows. Section 2 is devoted to notations, preliminary definitions, examples and results. In Section 3 we address the relaxation result and its consequences. In Section 4 we illustrate our model by showing experimental results on both synthetic and biological images.

\section{Definition AND MAin properties}

2.1. Distributional divergence and classical spaces. In the next subsection we recall the definition of the distributional space $L^{p, q}(\operatorname{Div} ; \Omega)$ and $\mathcal{D} \mathcal{M}^{p}(\Omega), 1 \leq p, q \leq+\infty$, (see $\left.[1,8]\right)$. In all the paper $\Omega \subset \mathbb{R}^{N}$ is an open bounded set with Lipschitz boundary. $N \geq 2$ is the space dimension. We denote by $\mathcal{A}(\Omega)$, the family of all open bounded subsets $A \subset \Omega$. $\mathcal{H}^{d}$ stands for the $d$-dimensional Haussdorf measure. Notation for Sobolev's and Lebesgue's spaces are standard.

Definition 2.1. For $U \in L^{p}\left(\Omega ; \mathbb{R}^{N}\right), 1 \leq p \leq+\infty$, set

$$
|\operatorname{Div} U|(\Omega):=\sup \left\{\int_{\Omega}\langle U, \nabla \varphi\rangle d x: \varphi \in C_{0}^{\infty}(\Omega),|\varphi| \leq 1\right\} .
$$

We say that $U$ is an $L^{p}$-divergence measure field, i.e. $U \in \mathcal{D} \mathcal{M}^{p}(\Omega)$, if

$$
\|U\|_{\mathcal{D} \mathcal{M}^{p}(\Omega)}:=\|U\|_{L^{p}\left(\Omega ; \mathbb{R}^{N}\right)}+|\operatorname{Div} U|(\Omega)<+\infty
$$

We recall that $U \in L^{p}\left(\Omega ; \mathbb{R}^{N}\right)$ belongs to $\mathcal{D} \mathcal{M}^{p}(\Omega)$ if and only if there exists a Radon measure denoted by $\operatorname{Div} U$ such that

$$
\int_{\Omega}\langle U, \nabla \varphi\rangle=-\int_{\Omega} \operatorname{DivU} \varphi \quad \forall \varphi \in C_{0}^{\infty}(\Omega),
$$

and the total variation of the measure $\operatorname{Div} U$ is given by $|\operatorname{Div} U|(\Omega)$.

We say that $U \in \mathcal{D} \mathcal{M}_{\text {loc }}^{p}(\Omega)$, if $U \in \mathcal{D} \mathcal{M}^{p}\left(\Omega^{\prime}\right)$ for every $\Omega^{\prime} \subset \subset \Omega$.

Let us recall the following result (see [9] Proposition 3.1).

Theorem 2.1. Let $\left\{U_{h}\right\}_{h} \subset \mathcal{D} \mathcal{M}^{p}(\Omega)$ be such that

$$
U_{h} \rightarrow U \quad \text { in } L^{p}\left(\Omega ; \mathbb{R}^{N}\right), \text { as } h \rightarrow+\infty \text { for } 1 \leq p<+\infty .
$$

Then

$$
\|U\|_{L^{p}\left(\Omega ; \mathbb{R}^{N}\right)} \leq \liminf _{h \rightarrow+\infty}\left\|U_{h}\right\|_{L^{p}\left(\Omega ; \mathbb{R}^{N}\right)}, \quad|\operatorname{Div} U|(\Omega) \leq \liminf _{h \rightarrow+\infty}\left|\operatorname{Div} U_{h}\right|(\Omega)
$$

Finally we define the following space

$$
\Delta \mathcal{M}_{\mathrm{loc}}^{p}(\Omega):=\left\{u \in W_{\mathrm{loc}}^{1, p}(\Omega), \nabla u \in \mathcal{D} \mathcal{M}_{\mathrm{loc}}^{p}(\Omega)\right\}
$$


2.2. p-capacity. If $\mathcal{K} \subset \mathbb{R}^{N}$ is a compact set and $\chi_{\mathcal{K}}$ denotes its characteristic function, we define:

$$
\operatorname{Cap}_{p}(\mathcal{K}, \Omega)=\inf \left\{\int_{\Omega}|\nabla f|^{p} d x, f \in C_{0}^{\infty}(\Omega), f \geq \chi_{\mathcal{K}}\right\}
$$

If $U \subset \Omega$ is an open set, its $p$-capacity is given by

$$
\operatorname{Cap}_{p}(U, \Omega)=\sup _{K \subset U} \operatorname{Cap}_{p}(K, \Omega) .
$$

Finally if $A \subset U \subset \Omega$ with $A$ Borel set and $U$ open, then

$$
\operatorname{Cap}_{p}(A, \Omega)=\inf _{A \subset U \subset \Omega} \operatorname{Cap}_{p}(U, \Omega) .
$$

We recall the following result (see for instance [17], Theorem 2.27) that explains the relationship between $p$-capacity and Hausdorff measures. Such a result is crucial to have geometric information on null $p$-capacity sets.

Theorem 2.2. Assume $1<p<N$. If $\mathcal{H}^{N-p}(A)<\infty$ then $\operatorname{Cap}_{p}(A, \Omega)=0$.

For general properties we refer the reader to $[13,17,23]$.

It is known (see [10]) that given a Radon measure $\mu$ the following decomposition holds

$$
\mu=\nu^{a}+\mu^{0}
$$

where the measure $\nu^{a}$ is absolutely continuous with respect to the $p$-capacity and $\mu^{0}$ is singular with respect to the $p$-capacity, that is concentrated on sets with zero $p$-capacity. Besides it is also known (see [10]) that every measure which is absolutely continuous with respect to the $p$-capacity can be characterized as an element of $L^{1}+W^{-1, p^{\prime}}$, leading to the finer decomposition:

$$
\mu=f d x-\operatorname{DivG}+\mu^{0}
$$

where $G \in L^{p^{\prime}}\left(\Omega ; \mathbb{R}^{2}\right)$ with $\frac{1}{p}+\frac{1}{p^{\prime}}=1$ and $f \in L^{1}(\Omega)$. In particular if $u \in \Delta \mathcal{M}^{p}(\Omega)$ by applying the classical Radon-Nikodym decomposition together with (2.4) to the measure Div $\nabla u$, denoted in this case with $\Delta u$, we get:

$$
\begin{aligned}
\operatorname{Div} \nabla u: & =\Delta u=\Delta u d x+\Delta^{s} u=\Delta u d x+\nu^{a}+\mu^{0}=\Delta u d x+f-\operatorname{DivG}+\mu^{0} \\
& =\Delta u d x-\operatorname{DivG}+\mu^{0}=\Delta \mathrm{udx}+\mu^{\mathrm{a}}+\mu^{0}
\end{aligned}
$$

where $f=0$, since we have applied decomposition (2.4) to the singular part $\Delta u^{s}$, with respect to the Lebesgue measure, of the measure $\Delta u$. To simplify the notation we have adopted the notation $\Delta u d x$ for the absolute continuous part of $\Delta u$. Moreover we have denoted by $\mu^{a}$ the measure - DivG which is absolutely continuous with respect to the $p$-capacity and also singular with respect to the Lebesgue measure.

2.3. Examples. In this subsection we briefly discuss some examples related to our model, in order to give an intuitive explanation of why the Laplacian operator is a right operator to restore point-like and curve-like singularities. 
Example 2.1. We begin with an example of point-like singularity. Let $\Omega=B_{1}(0) \subset \mathbb{R}^{2}$ be a ball centered at the origin with radius 1 . Let $B_{\epsilon}(0) \subset \mathbb{R}^{2}$ be a ball centered at the origin with radius $\epsilon$ such that $B_{\epsilon}(0) \subset \Omega$. Let $\delta_{0}$ be the Dirac's measure concentrated at the origin. Then we consider the solution of the Dirichlet problem:

$$
\begin{cases}-\Delta u_{\epsilon}=\delta_{0} & x \in B_{\epsilon}(0) \\ u_{\epsilon}=0 & x \in \partial B_{\epsilon}(0) .\end{cases}
$$

Suppose the image $I_{\epsilon} \in W_{0}^{1, p}(\Omega)$ is of the following form:

$$
I_{\epsilon}= \begin{cases}u_{\epsilon} & x \in B_{\epsilon}(0) \\ 0 & x \in \Omega \backslash B_{\epsilon}(0)\end{cases}
$$

We compute now

$$
\int_{B_{\epsilon}(0)}\left|\nabla I_{\epsilon}\right|^{p} d x=\int_{B_{\epsilon}(0)}\left|\nabla u_{\epsilon}\right|^{p} d x
$$

Let $q>p$ be such that $p q^{\prime}<2$, that is $q>\frac{2}{2-p}$. The by Holder inequality we have:

$$
\int_{B_{\epsilon}(0)}\left|\nabla u_{\epsilon}\right|^{p} d x \leq\left(\mathcal{L}^{2}\left(B_{\epsilon}(0)\right)\right)^{\frac{1}{q}}\left(\int_{B_{\epsilon}(0)}\left|\nabla u_{\epsilon}\right|^{p q^{\prime}} d x\right)^{\frac{1}{q^{\prime}}} .
$$

Since $u_{\epsilon}$ is the solution of problem, (2.6), classical a priori estimation (see [20] Theorem 9.1) ensures that

$$
\left\|\nabla u_{\epsilon}\right\|_{L^{s}\left(B_{\epsilon}(0)\right.} \leq C\left|\delta_{0}\right|\left(B_{\epsilon}(0)\right)=C<+\infty
$$

for all $s<2$ where the constant $C$ does not depend on $\epsilon$. Therefore by using (2.8) together with (2.7) by taking into account that $p q^{\prime}<2$, we get $\int_{B_{\epsilon}(0)}\left|\nabla I_{\epsilon}\right|^{p} \rightarrow 0$.

It means that the $L^{p}$-norm of the gradient is not sensitive to point-like singularities.

On the contrary, to the variation of the Laplacian measure in $R_{\epsilon}$ of $I_{\epsilon}$ is equivalent to compute the variation of the measure $\delta_{0}$. Therefore

$$
\left|\Delta I_{\epsilon}\right|\left(B_{\epsilon}(0)\right)=\sup _{\varphi \in C_{0}^{\infty}\left(B_{\epsilon}(0)\right)\|\varphi\|_{\infty} \leq 1} \int_{B_{\epsilon}(0)}|\varphi| d \delta_{0}=1
$$

It means that, the variation of the Laplacian measure, counts point-like singularities, which is precisely what is needed to preserve the singularities.

Example 2.2. We discuss an example of curve-like singularity. Let $\Omega=B_{1}(0) \subset \mathbb{R}^{2}$ be a ball centered at the origin with radius 1 . Let $\Gamma$ be the segment with parametrization $\alpha(t)=(t, 0)$ with $t \in\left[0, \frac{1}{2}\right]$. Let $\delta_{\Gamma}$ be the measure supported on $\Gamma$ defined in the following way: for every $A \subset \Omega$ Borel set

$$
\delta_{\Gamma}(A)=\int_{A \cap \Gamma} \alpha^{\prime}(t) d t=\mathcal{H}^{1}(A \cap \Gamma) .
$$

Let $R_{\epsilon} \subset \Omega$ be the rectangle $\left[0, \frac{1}{2}\right] \times[-\epsilon, \epsilon]$.

We consider the weak solution of the Dirichlet problem:

$$
\begin{cases}-\Delta u_{\epsilon}=\delta_{\Gamma} & x \in R_{\epsilon} \\ u_{\epsilon}=0 & x \in \partial R_{\epsilon} .\end{cases}
$$


As before we suppose to have an image $I_{\epsilon}$ given by:

$$
I_{\epsilon}= \begin{cases}u_{\epsilon} & x \in R_{\epsilon} \\ 0 & x \in \Omega \backslash R_{\epsilon}\end{cases}
$$

The same arguments of the previous example shows that

$$
\int_{R_{\epsilon}}\left|\nabla I_{\epsilon}(x)\right|^{p} d x \rightarrow 0 .
$$

It means that the $L^{p}$-norm of the gradient is not sensitive to curve-like singularities.

Next as in the example 2.1 we would like to compute the variation of the Laplacian measure.

Since $u_{\epsilon}$ is a weak solution of problem (2.9), we have that the variation of the Laplacian measure in $R_{\epsilon}$ coincides with the variation of the measure $\delta_{\Gamma}$, that is the length of the segment. Thus, by taking into account that $u_{\epsilon}=I_{\epsilon}$, we have

$$
\left|\Delta I_{\epsilon}\right|\left(R_{\epsilon}\right)=\mathcal{H}^{1}(\Gamma) .
$$

It means that Laplacian is sensitive to curve-like singularity.

2.4. Functionals and their properties. Let $f: \mathbb{R} \rightarrow[0,+\infty]$ and $g: \mathbb{R}^{N} \rightarrow[0,+\infty]$ be convex functions such that

$$
f(t) \leq C_{1}(1+|t|) \quad \forall t \in \mathbb{R}
$$

where $0<C_{1}<+\infty$ is a constant;

$$
g(\xi) \leq C_{2}\left(1+|\xi|^{p}\right) \quad \forall \xi \in \mathbb{R}^{N},
$$

where $0<C_{2}<+\infty$ is a constant.

We shall consider the following functionals for $u \in \Delta \mathcal{M}_{\mathrm{loc}}^{p}(\Omega)$ and $A \in \mathcal{A}(\Omega)$ :

$$
\begin{gathered}
F(u, A):= \begin{cases}\int_{A} f(\Delta u) d x+\int_{A} g(\nabla u) d x & \text { on } C^{2}(A), \\
+\infty & \text { on } \Delta \mathcal{M}_{\mathrm{loc}}^{p}(A) \backslash C^{2}(A) ;\end{cases} \\
\mathcal{F}(u, A):=\int_{A} f(\Delta u) d x+\int_{A} g(\nabla u) d x+\int_{A} f^{\infty}\left(\frac{d \mu^{a}}{d\left|\mu^{a}\right|}\right) d\left|\mu^{a}\right|+\int_{A} f^{\infty}\left(\frac{d \mu^{0}}{d\left|\mu^{0}\right|}\right) d\left|\mu^{0}\right|,
\end{gathered}
$$

where $f^{\infty}$ is the recession function given by $\lim _{s \rightarrow+\infty} \frac{f(s t)}{s}$, with $t \in \mathbb{R}$ and the measure $\mu^{a}$ and $\mu^{0}$ are given by decomposition (2.5). $\frac{d \mu^{a}}{d\left|\mu^{a}\right|}, \frac{d \mu^{0}}{d\left|\mu^{0}\right|}$ are the Radon-Nikodym derivatives of the measures $\mu^{a}$ and $\mu^{0}$ with respect to their total variation.

Finally we will always assume $1<p<\frac{N}{N-1}$. This restriction on $p$ is due to the fact that when $p \geq \frac{N}{N-1}$ the distributional divergence of $U$, and therefore the distributional Laplacian of a Sobolev function, cannot be a measure concentrated on sets with zero $p$-capacity (see [5]). We do this in order to allow concentration on sets of $N-2$ dimension such as points in $2 \mathrm{D}$ and curves in $3 \mathrm{D}$. Indeed in this case the support of $\mu^{a}=f-$ DivG contains sets of dimension $N-1$ such as $N-1$ manifolds with or without boundary inside $\Omega$ (see [23], Section 4.7, for a detailed discussion on the space $W^{-1, p^{\prime}}(\Omega)$ ), 
while the support of the term $\mu^{0}$ contains lower dimensional sets such as points for $N \geq 2$ or curves for $N \geq 3$.

2.5. Relaxation. Let $F$ be the functional defined in (2.12). For every $u \in \Delta \mathcal{M}_{\mathrm{loc}}^{p}(\Omega)$ we define the lower semicontinuous envelope or relaxed functional with respect to the $W_{\text {loc }}^{1, p}$-weak convergence of $F$ given by:

$$
S C^{-} F\left(u, \Omega^{\prime}\right):=\inf _{u_{h} \subset C^{2}\left(\Omega^{\prime}\right)}\left\{\liminf _{h \rightarrow+\infty} F\left(u_{h}, \Omega^{\prime}\right) \quad u_{h} \rightarrow u\right\},
$$

For the convenience of the reader we recall here that a sequence $\left\{u_{h}\right\} \subseteq W_{\text {loc }}^{1, p}(\Omega) W_{\text {loc }}^{1, p}$-weakly converges to $u \in W_{\text {loc }}^{1, p}(\Omega)$ if and only if for all $\varphi \in W^{1,-p^{\prime}}\left(\Omega^{\prime}\right)$ and $\Omega^{\prime} \subset \subset \Omega$ we have

$$
\left\langle u_{h}, \varphi\right\rangle_{W^{1, p}\left(\Omega^{\prime}\right) \times W^{1,-p^{\prime}}\left(\Omega^{\prime}\right)} \rightarrow\langle u, \varphi\rangle_{W^{1, p}\left(\Omega^{\prime}\right) \times W^{1,-p^{\prime}}\left(\Omega^{\prime}\right)} .
$$

We recall that if $G: \Delta \mathcal{M}_{\mathrm{loc}}^{p}(\Omega) \rightarrow \mathbb{R}$ is a continuous functional with respect to the $W_{\text {loc }}^{1, p}$-weak convergence we have:

$$
S C^{-}(F+G)=S C^{-} F+G .
$$

For general properties of the relaxation we refer to $[2,7]$.

\section{Relaxation theorem And existence of minimizers}

In this section we state and prove the relaxation formula. The result is close, in the spirit, to the the classical theorem of Goffmann-Serrin (see [16]) in the $B V$-framework. Indeed, in a similar way, to extend functional (2.12) to the whole space $\Delta \mathcal{M}_{\mathrm{loc}}^{p}(\Omega)$, the singular parts of the distributional divergence of $\nabla u$, have to be taken into account. To prove the relaxation argument we adapt the proof of ([16]) to our variational framework.

Theorem 3.1. Let $f: \mathbb{R} \rightarrow[0,+\infty), g: \mathbb{R}^{N} \rightarrow[0,+\infty)$ be convex functions satisfying (2.10) and (2.11). Then the following formula holds:

$$
S C^{-} F\left(u, \Omega^{\prime}\right)=\mathcal{F}\left(u, \Omega^{\prime}\right) \quad \forall u \in \Delta \mathcal{M}_{\mathrm{loc}}^{p}(\Omega), \quad \forall \Omega^{\prime} \subset \subset \Omega .
$$

Proof. Step one: We prove first the lower bound:

$$
\mathcal{F}\left(u, \Omega^{\prime}\right) \leq S C^{-} F\left(u, \Omega^{\prime}\right) \quad \forall u \in \Delta \mathcal{M}_{\mathrm{loc}}^{p}(\Omega), \quad \forall \Omega^{\prime} \subset \subset \Omega .
$$

Let $\left\{u_{h}\right\}_{h} \subset C^{2}\left(\Omega^{\prime}\right)$ such that $u_{h} \rightarrow u \in \Delta \mathcal{M}_{\text {loc }}^{p}(\Omega)$. Since $f$ is convex we have

$$
f(t)=\sup _{k}\left(a_{k}+b_{k} t\right), \quad f^{\infty}(t)=\sup _{k}\left(b_{k} t\right) \quad t \in \mathbb{R} .
$$

Then for every test function $\varphi \in C_{0}^{\infty}\left(\Omega^{\prime}\right)$ such that $0 \leq \varphi \leq 1$ we get

$$
F\left(u_{h}, \Omega^{\prime}\right) \geq \int_{\Omega^{\prime}} \varphi f\left(\Delta u_{h}\right) d x+\int_{\Omega^{\prime}} g\left(\nabla u_{h}\right) d x \geq \int_{\Omega^{\prime}} \varphi\left(a_{k}+b_{k} \Delta u_{h}\right) d x+\int_{\Omega^{\prime}} g\left(\nabla u_{h}\right) d x .
$$

By integrating by parts we obtain 


$$
F\left(u_{h}, \Omega^{\prime}\right) \geq \int_{\Omega^{\prime}} \varphi a_{k} d x-\int_{\Omega^{\prime}} b_{k} \nabla \varphi \nabla u_{h} d x+\int_{\Omega^{\prime}} g\left(\nabla u_{h}\right) d x
$$

Then taking the weak limit and integrating by parts we get

$$
\liminf _{h \rightarrow+\infty} F\left(u_{h}, \Omega^{\prime}\right) \geq \int_{\Omega^{\prime}} a_{k} \varphi d x+\int_{\Omega^{\prime}} b_{k} \varphi d \operatorname{Div} \nabla u+\int_{\Omega^{\prime}} g(\nabla u) d x
$$

hence by taking into account decomposition (2.5) we infer

$$
\liminf _{h \rightarrow+\infty} F\left(u_{h}, \Omega^{\prime}\right) \geq \int_{\Omega^{\prime}} \varphi\left(a_{k}+b_{k} \Delta u\right) d x+\int_{\Omega^{\prime}} \varphi b_{k} \frac{d \mu^{a}}{\left|d \mu^{a}\right|}\left|d \mu^{a}\right|+\int_{\Omega^{\prime}} \varphi b_{k} \frac{d \mu^{0}}{\left|d \mu^{0}\right|}\left|d \mu^{0}\right|+\int_{\Omega^{\prime}} g(\nabla u) d x .
$$

Finally, since the measures $d x, \mu_{a}$ and $\mu_{0}$ are mutually singular, to achieve inequality (3.2) it suffices to take the supremum over $\varphi$ and $k$ first, and then infimum over all the sequences $\left\{u_{h}\right\}_{h} \subset C^{2}\left(\Omega^{\prime}\right)$. So we get (3.2).

Step two : We now prove the upper bound $S C^{-} F\left(u, \Omega^{\prime}\right) \leq \mathcal{F}\left(u, \Omega^{\prime}\right)$ for every $u \in \Delta \mathcal{M}_{\text {loc }}^{p}(\Omega)$ and for all $\Omega^{\prime} \subset \subset \Omega$.

For $u \in \Delta \mathcal{M}_{\text {loc }}^{p}(\Omega)$, let $\left\{\tilde{u}_{h}\right\}_{h} \subset C^{\infty}\left(\Omega_{h}\right)$ be the mollified sequence of $u$ defined as $\tilde{u}_{h}:=u * \rho_{h}$ with $\left\{\rho_{h}\right\}_{h}$ a standard sequence of mollifiers and $\Omega_{h}=\left\{x \in \Omega\right.$ such that $\left.\operatorname{dist}(x, \partial \Omega)>\frac{1}{h}\right\}$.

For $x \in \Omega_{h}$ the support of $\rho_{h}(x-\cdot)$ is contained in $\Omega$, then by standard properties of the mollifying sequence, we have for $x \in \Omega_{h}$

$$
\begin{aligned}
& a_{k}+b_{k} \Delta \tilde{u}_{h}:=a_{k}+b_{k} \int_{\Omega} \rho_{h}(x-y) d \operatorname{Div} \nabla u \\
= & \int_{\Omega} \rho_{h}(x-y)\left(a_{k}+b_{k} \Delta u(y)\right) d y+\int_{\Omega} \rho_{h}(x-y) b_{k} \frac{d \mu^{a}}{\left|d \mu^{a}\right|}\left|d \mu^{a}\right|+\int_{\Omega} \rho_{h}(x-y) b_{k} \frac{d \mu^{0}}{\left|d \mu^{0}\right|}\left|d \mu^{0}\right| ;
\end{aligned}
$$

(for $x \in \Omega_{h}$ we can write the integral over all $\Omega$, since the integration with respect to $y$ is actually performed on the compact support of the kernel $\left.\rho_{h}(x-\cdot)\right)$.

By taking into account (3.3) we obtain

$$
\begin{aligned}
a_{k}+b_{k} \Delta \tilde{u}_{h} & \leq \int_{\Omega} \rho_{h}(x-y) f(\Delta u(y)) d y+\int_{\Omega} \rho_{h}(x-y) f^{\infty}\left(\frac{d \mu^{a}}{\left|d \mu^{a}\right|}\right)\left|d \mu^{a}\right| \\
& +\int_{\Omega} \rho_{h}(x-y) f^{\infty}\left(\frac{d \mu^{0}}{\left|d \mu^{0}\right|}\right)\left|d \mu^{0}\right| .
\end{aligned}
$$

Let $\Omega^{\prime} \subset \subset \Omega$ an open set. Since $\Omega_{h}$ is a sequence invading $\Omega$, we have for $h$ large enough $\Omega^{\prime} \subset \Omega_{h}$. Then for $h$ large enough by taking the supremum on $k$ on the left hand side of (3.4) and integrating over $\Omega^{\prime}$ with respect to $x$ we get:

$$
\begin{aligned}
\int_{\Omega^{\prime}} f\left(\Delta \tilde{u}_{h}\right) d x & \leq \int_{\Omega^{\prime}}\left(\int_{\Omega} \rho_{h}(x-y) f(\Delta u(y)) d y\right) d x+\int_{\Omega^{\prime}}\left(\int_{\Omega} \rho_{h}(x-y) f^{\infty}\left(\frac{d \mu^{a}}{\left|d \mu^{a}\right|}\right)\left|d \mu^{a}\right|\right) d x \\
& +\int_{\Omega^{\prime}}\left(\int_{\Omega} \rho_{h}(x-y) f^{\infty}\left(\frac{d \mu^{0}}{\left|d \mu^{0}\right|}\right)\left|d \mu^{0}\right|\right) d x
\end{aligned}
$$

By applying, thanks to (2.10), Fubini's Theorem to the right hand side of (3.5) we have

$$
\int_{\Omega^{\prime}}\left(\int_{\Omega} \rho_{h}(x-y) f(\Delta u(y)) d y\right) d x=\int_{\Omega^{\prime}} f(\Delta u(y))\left(\int_{\Omega} \rho_{h}(x-y) d x\right) d y
$$


and then

$$
\begin{aligned}
& \int_{\Omega^{\prime}}\left(\int_{\Omega} \rho_{h}(x-y) f^{\infty}\left(\frac{d \mu^{a}}{d\left|\mu^{a}\right|}\right) d\left|\mu^{a}\right|\right) d x=\int_{\Omega^{\prime}} f^{\infty}\left(\frac{d \mu^{a}}{d\left|\mu^{a}\right|}\right)\left(\int_{\Omega} \rho_{h}(x-y) d x\right) d\left|\mu^{a}\right|, \\
& \int_{\Omega^{\prime}}\left(\int_{\Omega} \rho_{h}(x-y) f^{\infty}\left(\frac{d \mu^{0}}{d\left|\mu^{0}\right|}\right) d\left|\mu^{0}\right|\right) d x=\int_{\Omega^{\prime}} f^{\infty}\left(\frac{d \mu^{0}}{d\left|\mu^{0}\right|}\right)\left(\int_{\Omega} \rho_{h}(x-y) d x\right) d\left|\mu^{0}\right|,
\end{aligned}
$$

$$
\int_{\Omega^{\prime}} f\left(\Delta \tilde{u}_{h}\right) d x \leq \int_{\Omega^{\prime}} f(\Delta u) d x+\int_{\Omega^{\prime}} f^{\infty}\left(\frac{d \mu^{a}}{d\left|\mu^{a}\right|}\right) d\left|\mu^{a}\right|+\int_{\Omega^{\prime}} f^{\infty}\left(\frac{d \mu^{0}}{d\left|\mu^{0}\right|}\right)\left|d \mu^{0}\right|,
$$

where we have used the fact that $\int_{\Omega} \rho_{h}(x-y) d x \leq \int_{\mathbb{R}^{N}} \rho_{h}(x-y) d x=1$.

By the same argument we get also that

$$
\int_{\Omega^{\prime}} g\left(\nabla \tilde{u}_{h}\right) d x \leq \int_{\Omega^{\prime}} g(\nabla u) d x .
$$

Then (3.6) and (3.7) imply

$$
\int_{\Omega^{\prime}} f\left(\Delta \tilde{u}_{h}\right) d x+\int_{\Omega^{\prime}} g\left(\nabla \tilde{u}_{h}\right) d x \leq \mathcal{F}\left(u, \Omega^{\prime}\right) .
$$

Therefore since $\left\{\tilde{u}_{h}\right\}_{h} \subset C^{2}\left(\Omega^{\prime}\right)$ and $\tilde{u}_{h} \rightarrow u$ with respect to the $W_{\text {loc }}^{1, p}(\Omega)$ convergence, we have by taking into account (3.8)

$$
\begin{aligned}
S C^{-} F\left(u, \Omega^{\prime}\right) & =\inf _{u_{h} \subset C^{2}\left(\Omega^{\prime}\right)}\left\{\liminf _{h \rightarrow+\infty} F\left(u_{h}, \Omega^{\prime}\right) \quad u_{h} \rightarrow u\right\} \\
& \leq \liminf _{h \rightarrow+\infty} F\left(\tilde{u}_{h}, \Omega^{\prime}\right) \leq \mathcal{F}\left(u, \Omega^{\prime}\right),
\end{aligned}
$$

and therefore, being $\Omega^{\prime}$ arbitrary, the thesis is achieved.

\section{Numerical Applications}

In view of numerical applications we will always consider $N=2, f(t)=|t|$ and $g(\xi)=\frac{1}{p}|\xi|^{p}$ (with $1<p<2$ ), so that the recession function $f^{\infty}(t)$ is equal to $|t|$. Moreover we add an $L^{2}$-discrepancy term, and we consider the following functionals:

$$
J\left(u, \Omega^{\prime}\right):= \begin{cases}\int_{\Omega^{\prime}}|\Delta u| d x+\frac{1}{p} \int_{\Omega^{\prime}}|\nabla u|^{p} d x+\frac{\lambda}{2} \int_{\Omega^{\prime}}\left|u-u_{0}\right|^{2} d x & \text { on } C^{2}(\Omega), \\ +\infty & \text { on } \Delta \mathcal{M}_{\mathrm{loc}}^{p}(\Omega) \backslash C^{2}(\Omega),\end{cases}
$$

and for all $\Omega^{\prime} \subset \subset \Omega$

$$
\mathcal{J}\left(u, \Omega^{\prime}\right):=\int_{\Omega^{\prime}}|\Delta u| d x+\frac{1}{p} \int_{\Omega^{\prime}}|\nabla u|^{p} d x+\left|\mu^{a}\right|\left(\Omega^{\prime}\right)+\left|\mu^{0}\right|\left(\Omega^{\prime}\right)+\frac{\lambda}{2} \int_{\Omega^{\prime}}\left|u-u_{0}\right|^{2} d x,
$$

where $u_{0} \in L^{2}\left(\Omega^{\prime}\right)$ is the observed data, and $\lambda$ is a positive weight.

Let us prove the existence and uniqueness of a minimum for functional $\mathcal{J}$, before that we focus on discrete analysis.

Proposition 4.1. There exists a unique minimum $u \in \Delta \mathcal{M}_{\mathrm{loc}}^{p}(\Omega)$ of functional $\mathcal{J}$ defined in (4.2). Moreover the following equality holds for all $\Omega^{\prime} \subset \subset \Omega$

$$
\inf _{u \in C^{2}(\Omega)} J\left(u, \Omega^{\prime}\right)=\inf _{u \in \Delta \mathcal{M}_{\mathrm{loc}}^{p}(\Omega)} J\left(u, \Omega^{\prime}\right)=\min _{u \in \Delta \mathcal{M}_{\mathrm{loc}}^{p}(\Omega)} \mathcal{J}\left(u, \Omega^{\prime}\right)
$$


Proof. Let $\left\{u_{h}\right\}_{h} \subset \Delta \mathcal{M}_{\text {loc }}^{p}(\Omega)$ be a minimizing sequence of $\mathcal{J}$. Then it follows that

$$
\left\|u_{h}\right\|_{L_{\mathrm{loc}}^{p}(\Omega)}^{p}+\left\|\nabla u_{h}\right\|_{W_{\mathrm{loc}}^{1, p}(\Omega)}^{p} \leq M
$$

Therefore, there exists a subsequence, still denoted by $\left\{u_{h}\right\}_{h} \subset \Delta \mathcal{M}_{\text {loc }}^{p}(\Omega)$, and a function $u \in W_{l o c}^{1, p}(\Omega)$ such that $u_{h} \rightarrow u$ with respect to the $W_{l o c}^{1, p}(\Omega)$-weak convergence. From Theorem 2.1 we have for all $\Omega^{\prime} \subset \subset \Omega$

$$
|\operatorname{Div} \nabla u|\left(\Omega^{\prime}\right) \leq \liminf _{h \rightarrow+\infty} \mathcal{J}\left(u_{h}, \Omega^{\prime}\right) \leq M,
$$

which implies $u \in \Delta \mathcal{M}_{\text {loc }}^{p}(\Omega)$. As immediate consequence of Theorem 3.1 and property (2.15), functional $\mathcal{J}$ is lower semicontinuous with respect to the $W_{\text {loc }}^{1, p}$-weak convergence. Then the existence of a minimum follows via the direct methods of the calculus of variations, while the uniqueness is consequence of the strong convexity of $\mathcal{J}$.

Finally property (4.3) can be achieved by standard arguments (see for instance [7]).

4.1. Discrete setting. We define the discrete rectangular domain $\Omega$ of step size $\delta x=1$ and dimension $d_{1} d_{2}$, where $\Omega=\left\{1, \ldots, d_{1}\right\} \times\left\{1, \ldots, d_{2}\right\} \subset \mathbb{Z}^{2}$. In order to simplify the notations we set $X=R^{d_{1} \times d_{2}}$ and $Y=X \times X . u \in X$ denotes a matrix of size $d_{1} \times d_{2}$. For $u \in X, u_{i, j}$ denotes its (i,j)-th component with $(i, j) \in\left\{1, \ldots, d_{1}\right\} \times\left\{1, \ldots, d_{2}\right\}$. For $g \in Y, g_{i, j}$ denotes the $\mathrm{i}, \mathrm{j}$-th component of $g$ with $g_{i, j}=\left(g_{i, j}^{1}, g_{i, j}^{2}\right)$ and $(i, j) \in\left\{1, \ldots, d_{1}\right\} \times\left\{1, \ldots, d_{2}\right\}$

We endowed the space $X$ and $Y$ with standard scalar product and standard norm. For $u, v \in X$ we have:

$$
\langle u, v\rangle_{X}=\sum_{i=1}^{d_{1}} \sum_{j=1}^{d_{2}} u_{i, j} v_{i, j}
$$

For $g, h \in Y$ we have:

$$
\langle g, h\rangle_{Y}=\sum_{i=1}^{d_{1}} \sum_{j=1}^{d_{2}} \sum_{l=1}^{2} g_{i, j}^{l} h_{i, j}^{l} .
$$

For $u \in X$ and $p \in[1,+\infty)$ we set:

$$
|u|_{p}:=\left(\sum_{i=1}^{d_{1}} \sum_{j=1}^{d_{2}}\left|u_{i, j}\right|^{p}\right)^{\frac{1}{p}}
$$

For $g \in Y$ and $p \in[1,+\infty)$ :

$$
\|g\|_{p}:=\left(\sum_{i=1}^{d_{1}} \sum_{j=1}^{d_{2}}\left|g_{i, j}\right|_{2}^{p}\right)^{\frac{1}{p}} .
$$

If $G, F$ are two vector spaces and $H: G \rightarrow F$ is a linear operator, then the norm of $H$ is defined by

$$
\|H\|:=\max _{\|u\|_{G} \leq 1}\left(\|H u\|_{F}\right) .
$$

Definition 4.1. A function $F: X \rightarrow \mathbb{R}$ is said to be L-lipschitz differentiable if it is differentiable and

$$
|\nabla F(u)-\nabla F(v)|_{2} \leq L|u-v|_{2},
$$

for every $u, v \in X$. 
Definition 4.2. Let $\psi: X \rightarrow \mathbb{R}$ be a convex function. The operator

$$
\operatorname{prox}_{\psi}: X \rightarrow X \quad x \longmapsto \arg \min _{y \in X}\left\{\psi(y)+\frac{1}{2}|y-x|_{2}^{2}\right\}
$$

is called proximal operator associated to $\psi$.

If $\operatorname{prox}_{\lambda \psi}$ can be computed exactly for every $\lambda \geq 0$ and every $x \in X$, the function $\psi$ is said to be simple.

If $u \in X$ the gradient $\nabla u \in Y$ is given by:

$$
(\nabla u)_{i, j}=\left((\nabla u)_{i, j}^{1},(\nabla u)_{i, j}^{2}\right)
$$

where

$$
\begin{gathered}
(\nabla u)_{i, j}^{1}= \begin{cases}u_{i+1, j}-u_{i, j} & \text { if } i<d_{1} \\
0 & \text { if } i=d_{1}\end{cases} \\
(\nabla u)_{i, j}^{2}= \begin{cases}u_{i, j+1}-u_{i, j} & \text { if } j<d_{2} \\
0 & \text { if } j=d_{2} .\end{cases}
\end{gathered}
$$

We also introduce the discrete version of the divergence operator defined as the adjoint operator of the gradient: $\operatorname{div}=-\nabla^{*}$. If $p \in Y$, we have

$$
(\operatorname{div} p)_{i, j}= \begin{cases}p_{i, j}^{1}+p_{i, j}^{2} & \text { if } i, j=1 \\ p_{i, j}^{1}+p_{i, j}^{2}-p_{i, j-1}^{2} & \text { if } i=1,1<j<d_{2} \\ p_{i, j}^{1}-p_{i-1, j}^{1}+p_{i, j}^{2}-p_{i, j-1}^{2} & \text { if } 1<i<d_{1}, 1<j<d_{2} \\ -p_{i-1, j}^{1}+p_{i, j}^{2}-p_{i, j-1}^{2} & \text { if } i=d_{1}, 1<j<d_{2} \\ p_{i, j}^{1}-p_{i-1, j}^{1}+p_{i, j}^{2} & \text { if } 1<i<d_{1}, j=1 \\ p_{i, j}^{1}-p_{i-1, j}^{1}-p_{i, j-1}^{2} & \text { if } 1<i<d_{1}, j=d_{2} \\ -\left(p_{i-1, j}^{1}+p_{i, j-1}^{2}\right) & \text { if } i=d_{1}, j=d_{2} .\end{cases}
$$

Then we can define the discrete version of the Laplacian operator as $\Delta u=\operatorname{div}(\nabla u)$.

4.2. Nesterov scheme. Here we briefly recall the fast descent gradient Nesterov's algorithm (see $[18,19])$. We state it in the formulation proposed in [21, 22]. For further details and general statements we refer the reader to $[21,22]$ and references therein.

Proposition 4.2. Let $F: X \rightarrow \mathbb{R}$ be given by:

$$
F(u)=F_{1}(u)+F_{2}(u) \quad \text { for } u \in X
$$


where $F_{1}$ is a convex L-Lipschitz differentiable function and $F_{2}$ a simple function. Then the following algorithm ${ }^{3}$ :

$$
\left\{\begin{array}{l}
u_{0} \in X \quad A_{0}=0 \quad g=0 \quad u=0 \\
\quad d o \text { for } k: 1, \ldots, K \\
t=\frac{2}{L} \\
a=t+\sqrt{t^{2}+4 t A} \\
v=\operatorname{prox}_{A F_{2}}\left(u_{0}-g\right) \\
y=\frac{A u+A v}{A+a} \\
u=\operatorname{prox}_{\frac{1}{L} F_{2}}\left(y-\frac{1}{L} \nabla F_{1}(y)\right) \\
g=g+a \nabla F_{1}(u) \\
A=A+a
\end{array}\right.
$$

ensures that:

$$
0 \leq F\left(u_{k}\right)-F\left(u^{*}\right) \leq L \frac{\left|u^{*}-u_{0}\right|_{2}^{2}}{k^{2}},
$$

where $u^{*} \in X$ is a minimum point of $F$ and $u_{0} \in X$ is an initial data.

Remark 4.1. In order to apply the previous algorithm it is crucial to compute exactly the proximal operator prox $\alpha_{\alpha F_{2}}$ for every $\alpha \in \mathbb{R}^{+}$. This is the case since by assumption $F_{2}$ is a simple function.

4.3. The discrete functionals. For $u \in X$ we define the discrete version of functional (4.1)

$$
J(u)=\|\Delta u\|_{1}+\frac{1}{p}\|\nabla u\|_{p}^{p}+\frac{\lambda}{2}\left|u-u_{0}\right|_{2}^{2} .
$$

We also introduce its smoother counterpart given by:

$$
J_{\epsilon}(u)=\sum_{i=1}^{d_{1}} \sum_{j=1}^{d_{2}} w_{\epsilon}\left(\left|(\Delta u)_{i, j}\right|\right)+\frac{1}{p} \sum_{i=1}^{d_{1}} \sum_{j=1}^{d_{2}} w_{\epsilon}\left(\left|(\nabla u)_{i, j}\right|^{p}\right)+\frac{\lambda}{2} \sum_{i=1}^{d_{1}} \sum_{j=1}^{d_{2}}\left|u_{i, j}-\left(u_{0}\right)_{i, j}\right|^{2},
$$

where $\epsilon>0$ is a small fixed parameter and $w_{\epsilon}$ is the Huber function given by:

$$
w_{\epsilon}(x)= \begin{cases}|x| & \text { if }|x| \geq \epsilon \\ \frac{x^{2}}{2 \epsilon}+\frac{\epsilon}{2} . & \end{cases}
$$

We shall consider the minimization problems:

$$
\begin{aligned}
& \min _{u \in X} J(u), \\
& \min _{u \in X} J_{\epsilon}(u) .
\end{aligned}
$$

As in [22] we define the notion of $\delta$-solution associated to problem (4.8), which will be used to give an estimation of the number of iterations of the minimization algorithm.

Definition 4.3. A $\delta$-solution of (4.8) is an element $u_{\delta} \in X$ such that

$$
J\left(u_{\delta}\right)-J(\bar{u}) \leq \delta,
$$

where $\bar{u}$ is a solution of problem (4.8).

\footnotetext{
${ }^{3}$ we omit the dependance on $k$ to simplify the notation
} 
4.4. The proposed algorithm. We minimize $J_{\epsilon}$ by applying algorithm (4.4) with $F_{1}(u)=J_{\epsilon}(u)=\sum_{i=1}^{d_{1}} \sum_{j=1}^{d_{2}} w_{\epsilon}\left(\left|(\Delta u)_{i, j}\right|\right)+\frac{1}{p} \sum_{i=1}^{d_{1}} \sum_{j=1}^{d_{2}} w_{\epsilon}\left(\left|(\nabla u)_{i, j}\right|^{p}\right)+\frac{\lambda}{2} \sum_{i=1}^{d_{1}} \sum_{j=1}^{d_{2}}\left|u_{i, j}-\left(u_{0}\right)_{i, j}\right|^{2} \quad F_{2}(u)=0$. Indeed it is not difficult to check that

$$
\nabla F_{1}(u)=\Delta(\Psi)-\operatorname{div}(\Phi)+\lambda\left(u-u_{0}\right)
$$

where

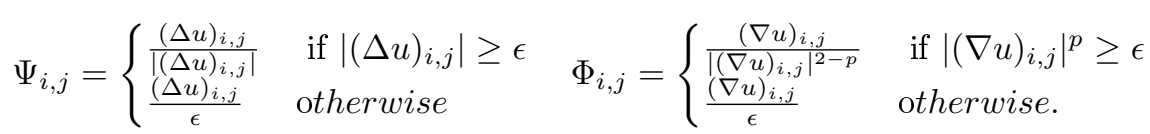

Then, taking into account that $\Psi$ and $\Phi$ are Lipschitz functions with constant $\frac{1}{\epsilon}$ and $\frac{1}{\epsilon^{p}}$ respectively, we infer

$$
\left|\nabla F_{1}(u)-\nabla F_{1}(v)\right|_{2} \leq\left(\frac{\|\Delta\|_{2}^{2}}{\epsilon}+\frac{\|\operatorname{div}\|_{2}^{2}}{\epsilon^{2-p}}+\lambda\right)|u-v|_{2}
$$

Therefore, by recalling that, if $N=2,\|\Delta\|_{2} \leq 8$ and $\|\operatorname{div}\|_{2} \leq 2 \sqrt{2}$, we conclude that

$$
\left|\nabla F_{1}(u)-\nabla F_{1}(v)\right|_{2} \leq\left(\frac{64}{\epsilon}+\frac{8}{\epsilon^{2-p}}+\lambda\right)|u-v|_{2} .
$$

Thanks to inequality (4.11) we are in position of applying algorithm (4.4). Then in our case algorithm (4.4) ensures that:

$$
0 \leq J_{\epsilon}\left(u_{k}\right)-J_{\epsilon}\left(u_{\epsilon}^{*}\right) \leq\left(\frac{64}{\epsilon}+\frac{8}{\epsilon^{2-p}}+\lambda\right) \frac{\left|u_{\epsilon}^{*}-u_{0}\right|_{2}^{2}}{k^{2}}
$$

where $u_{\epsilon}^{*}$ is a minimum of $J_{\epsilon}$.

4.5. Computer examples. Before running our algorithm all the parameters have to be fixed. It is easy to see that for every $u \in X$ we have

$$
0 \leq J_{\epsilon}(u)-J(u) \leq d_{1} d_{2} \epsilon
$$

Then by using (4.13) (4.12) and the fact that $u_{\epsilon}^{*}$ is a minimum of $J_{\epsilon}$ we have

$$
J\left(u_{k}\right) \leq J_{\epsilon}\left(u_{k}\right) \leq J_{\epsilon}\left(u_{\epsilon}^{*}\right)+\left(\frac{64}{\epsilon}+\frac{8}{\epsilon^{2-p}}+\lambda\right) \frac{\left|u_{\epsilon}^{*}-u_{0}\right|_{2}^{2}}{k^{2}} \leq J_{\epsilon}(\bar{u})+\left(\frac{64}{\epsilon}+\frac{8}{\epsilon^{2-p}}+\lambda\right) \frac{\left|u_{\epsilon}^{*}-u_{0}\right|_{2}^{2}}{k^{2}},
$$

where $\bar{u}$ is a minimum of $J$. By applying again bound (4.13) we deduce

$$
J\left(u_{k}\right) \leq J(\bar{u})+d_{1} d_{2} \epsilon+\left(\frac{64}{\epsilon}+\frac{8}{\epsilon^{2-p}}+\lambda\right) \frac{\left|u_{\epsilon}^{*}-u_{0}\right|_{2}^{2}}{k^{2}} .
$$

Therefore the worst case precision to get a $\delta$-solution of (4.8) is:

$$
J\left(u_{k}\right)-J(\bar{u})=\left(\frac{64}{\epsilon}+\frac{8}{\epsilon^{2-p}}+\lambda\right) \frac{\left|\bar{u}-u_{0}\right|_{2}^{2}}{k^{2}}+d_{1} d_{2} \epsilon ;
$$

then the optimal choices are

$$
\left.\epsilon=\frac{\delta}{d_{1} d_{2}}, \quad K=\left[\sqrt{\left(\frac{64 d_{1} d_{2}}{\delta}+8 \frac{d_{1} d_{2}}{\delta^{2-p}}+\lambda\right)}\right] C\right]+1,
$$

where $C:=\max _{X}\left|\bar{u}-u_{0}\right|_{2}$ and $K$ the total number of iterations. For images rescaled in $[0,1]$, in the worst case problem we deal with (see Figure 5 ), the number of iterations $K$ needed to get a $\delta$-solution of order 1 does not exceed the value 30000. In all numerical tests we let run the algorithm for no more 
than 2000 iterations. The parameter $\epsilon$ is always fixed in order to get a $\delta$-solution of order 1 . This choice seems to lead to good restoration results.

The parameter $\lambda$ is tuned according to the level noise and its value is specified on each numerical test.

Finally as exponent $p$ we always take $p=1.5$.

4.6. A model synthetic example. In Figure 1 we build up an example of ideal original image $u$ we have in mind. Such an image must mimic the solution of the problem:

$$
\begin{cases}-\Delta u=\mu, & \text { on } B \\ u=0 & \text { on } \partial B,\end{cases}
$$

where $\mu$ can be a measure concentrate on curves or points and $B$ is a suitable neighborhood of the support of the measure $\mu$.

For instance if for $i=1, \ldots, l \Gamma_{i}$ is an open line, we consider the image $\delta_{\Gamma_{i}}$

$$
\begin{cases}\delta_{\Gamma_{i}}(i, j)=1 & \text { if }(i, j) \in \Gamma_{i} \\ 0 & \text { otherwise }\end{cases}
$$

We fix a window $W_{i}$ containing $\Gamma_{i}$. We consider the Dirichlet problem

$$
\begin{cases}-\Delta v_{i}=\delta_{\Gamma_{i}}, & \text { on } W_{i} \\ v_{i}=0 & \text { on } \partial W_{i},\end{cases}
$$

To compute $v_{i}$ we minimize via classical descent gradient the following functional:

$$
F\left(v_{i}\right)=\frac{1}{2}\left|\nabla v_{i}\right|^{2}-\delta_{\Gamma_{i}} v_{i} .
$$

Indeed, if $v_{i}$ is a minimum we have that

$$
0=\nabla F\left(v_{i}\right)=-\Delta v_{i}-\delta_{\Gamma_{i}} .
$$

Moreover $F$ is Lipschitz differentiable with Lipschitz constant $L \leq\|\Delta\|_{2} \leq 8$. In order obtain a minimum $v_{i}$ we iterate the standard procedure:

$$
\left\{\begin{array}{l}
v_{i}^{n+1}=v_{i}^{n}-d t \nabla F\left(v_{i}^{n}\right) \\
v^{i}(0)=0
\end{array}\right.
$$

with $d t \leq \frac{1}{L}$ (we consider $d t=\frac{1}{10}$ ). Thus $\lim _{n \rightarrow+\infty} v_{i}^{n}=v_{i}$, with $v_{i}$ minimum of $F$. Then we extend $v_{i}$ as 0 outside $W$. So we have :

$$
u_{i}= \begin{cases}v_{i} & \text { in } W_{i} \\ 0 & \text { otherwise. }\end{cases}
$$

We repeat this procedure for every $i$ and we define the ideal original image as

$$
u=\sum_{i=1}^{l} u_{i}
$$

In such a way we have a synthetic image $u$ which locally behaves as the solution of Dirichlet problem (4.14) (see Figure 1). 


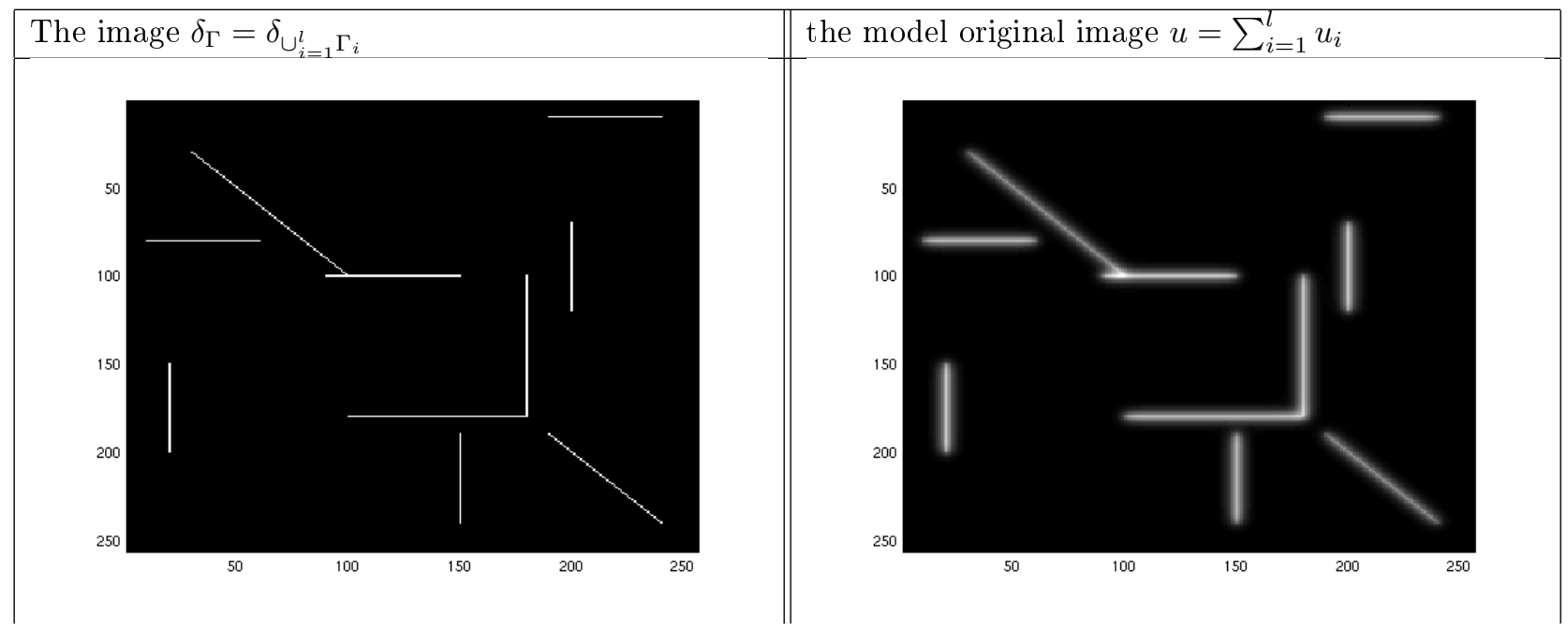

Figure 1. Synthetic images. The construction of the ideal original image. Top left: the image of filaments $\delta_{\Gamma}$. Top right: the image $u$, that we consider as original image.

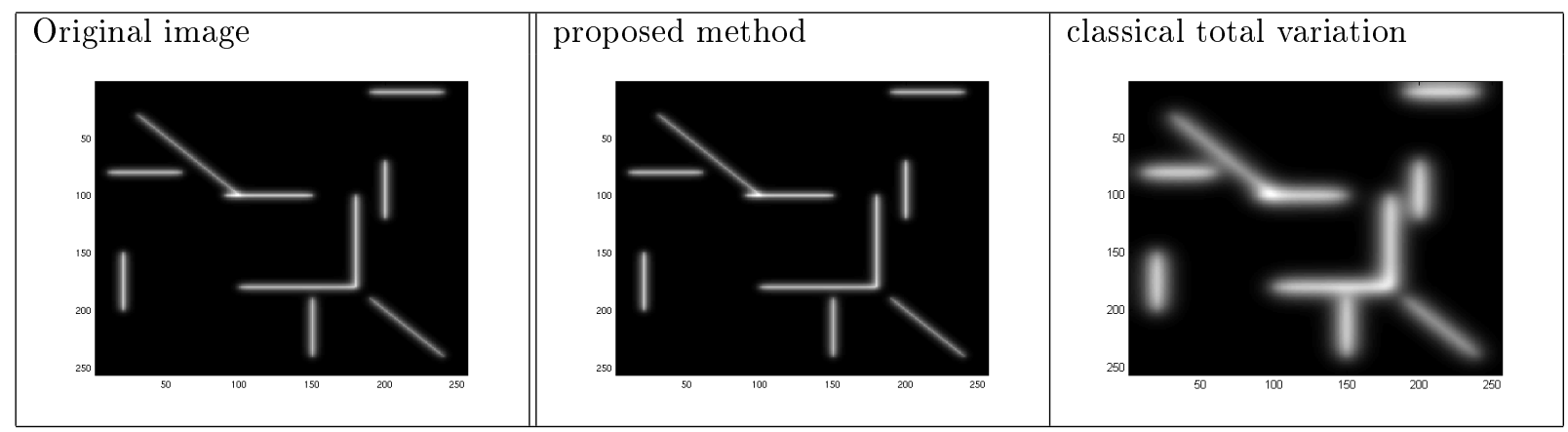

Figure 2. Synthetic images. Left bottom: original image. Center bottom: restored image by using our method. Right bottom: restored image by using classical total variation minimization. Since the gradient is not sensitive to curve-like singularities, the total variation does not preserve such singularities and some filaments tends to disappear.

In figure 2 we compare our method with the classical total variation minimization one, in order to verify that the minimization of the proposed functional performs a better preservation of curve-like singularities. In 3 and 4 we test the algorithm against noise on the ideal synthetic image $u$. A Gaussian Noise is added to the original image. The image domain is of size $d_{1} \times d_{2}=256 \times 256$. CPU time is about 40 s running on an Intel (R) Xeon(R) CPU 5120 at $1.86 \mathrm{GHz}$.

4.7. Test on real images. Here we test our model on real images. We assume that these image behave like the ideal image $u_{0}$ in previous section.

Figure 5 shows an application of the algorithm on a biological image of cell spots provided for us by "Institut Pasteur de Paris". The image domain is of size $d_{1} \times d_{2}=757 \times 510$. We always fix 


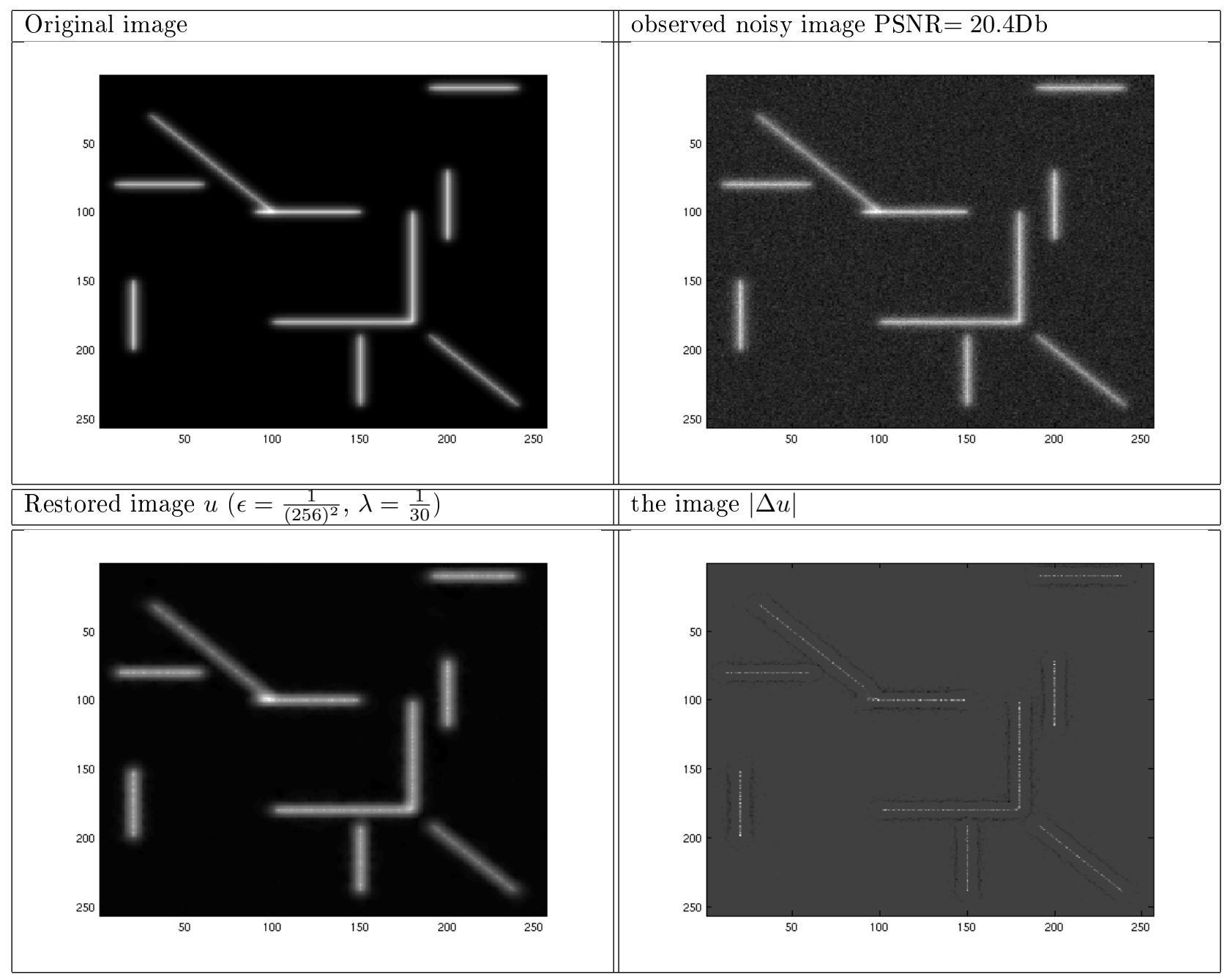

Figure 3. Synthetic images. Top left: Original images. Top right: noisy image. Down left: Restored image given by a $\delta$-solution of order 1. Down right: The support of $|\Delta u|$, where $u$ is the restored image. The curve like-singularities are preserved in the denoising process

$\epsilon=\frac{1}{757 \times 510}$. We let run the algorithm until 2000 iterations to obtain a $\delta$-solution of order 1 shown in Figure 6. CPU time is about $5 \mathrm{mn}$ running on an Intel (R) Xeon(R) CPU 5120 at $1.86 \mathrm{GHz}$.

Finally in Figure 8 we test our model on a real noisy image which contains spots and curves. The image domain is of size $d_{1} \times d_{2}=570 \times 560$. CPU time is about $7 \mathrm{mn}$ running on an Intel (R) Xeon(R) CPU 5120 at $1.86 \mathrm{GHz}$. 


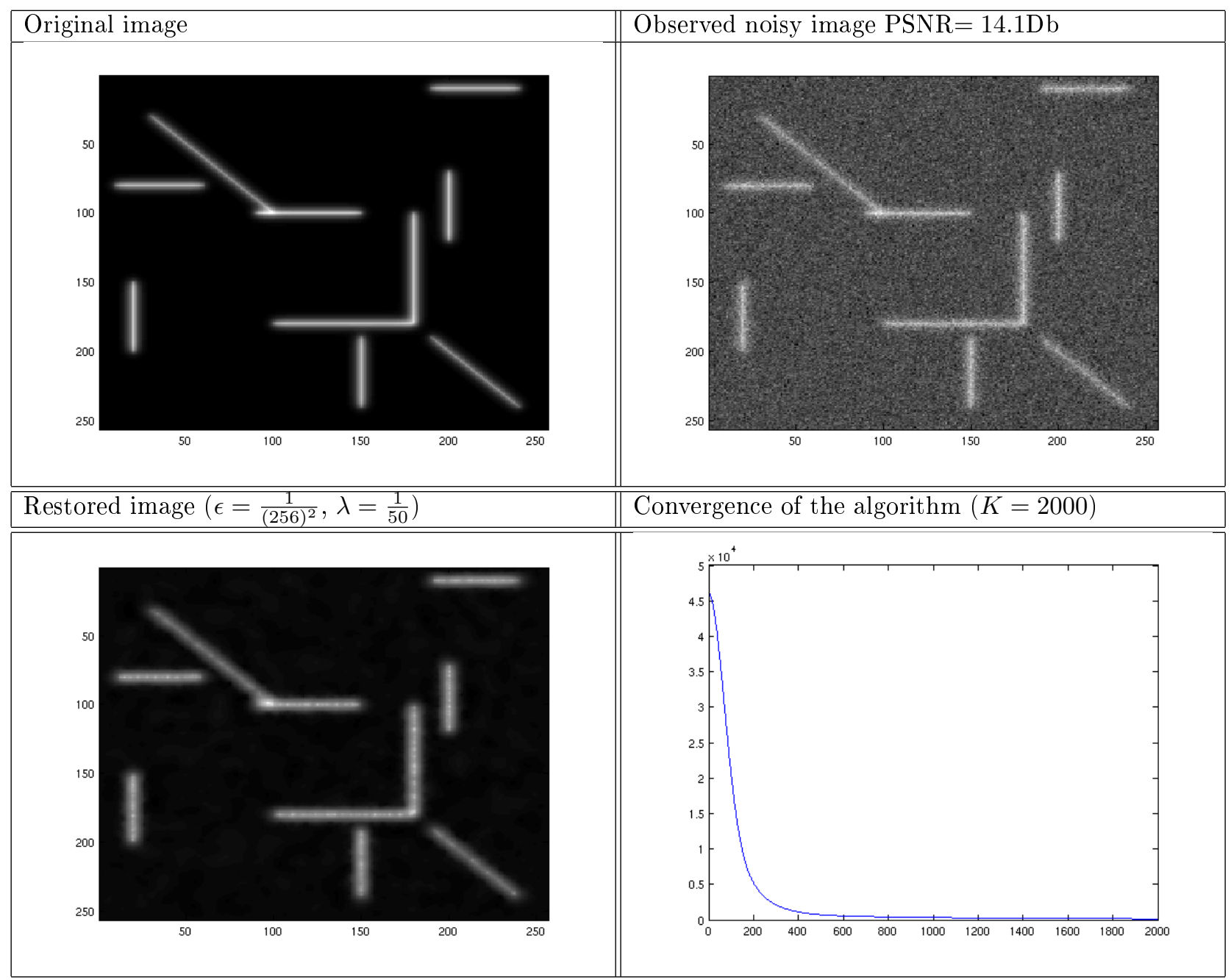

Figure 4. Synthetic images. We test the proposed algorithm on noisy data. Top left: Original image. Top right: noisy image. Down left: Restored image given by a $\delta$-solution of order 1 . Down right: Convergence on the algorithm. On the $y$-axis the value of $J\left(u_{k}\right)$. On the $x$-axis the number of iterations. 


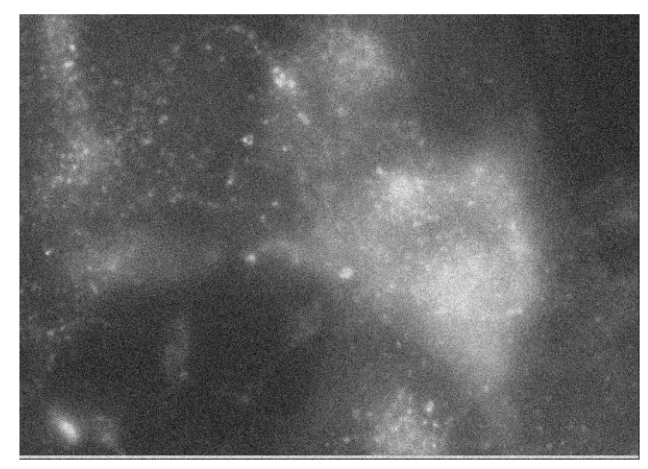

FiguRE 5. Noisy image of cell spots.

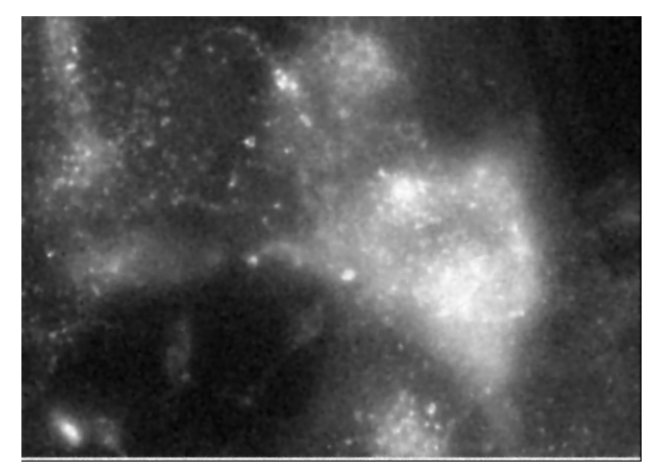

FiguRE 6 . Restored image given by a $\delta$-solution of order 1 . Number of iterations $K=2000, \lambda=\frac{1}{20}$. since the level of noise is not too high and the intensity of the images on spots is elevated, the algorithm is capable to restore the image by preserving point-like structures. 


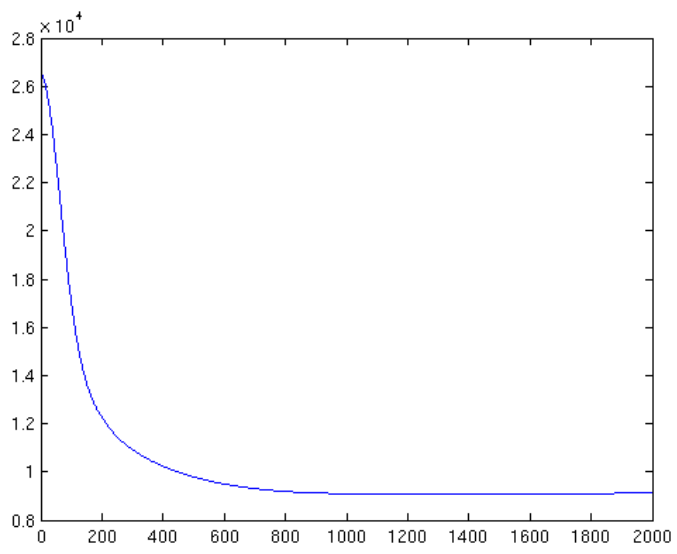

Figure 7. Convergence of the algorithm. On the $y$-axis the value of $J\left(u_{k}\right)$. On the $x$-axis the number of iterations.

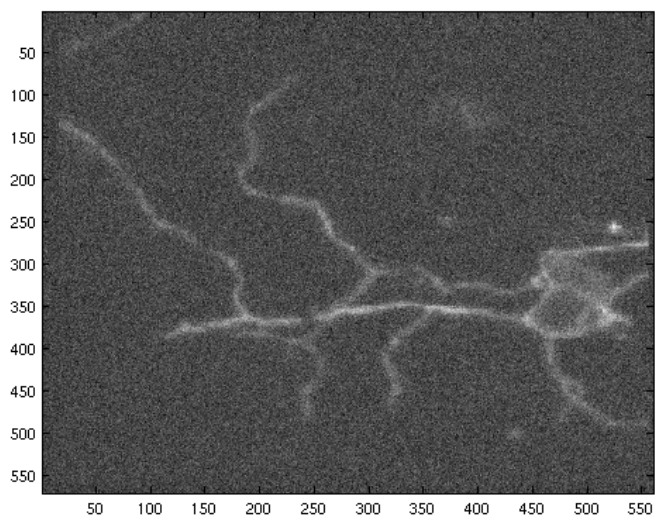

FiguRE 8. Real noisy image: we test our algorithm on a real image of a blood vessels network corrupted by Gaussian noise. Image size $d_{1} \times d_{2}=560 \times 570$. 


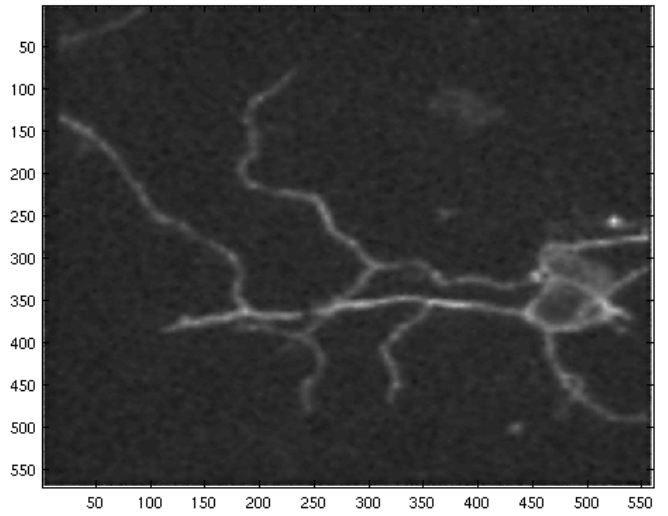

Figure 9. Restored image: $\delta=1, \lambda=\frac{1}{100}, \epsilon=\frac{1}{560 \times 570}$. Number of iterations $K=2000$

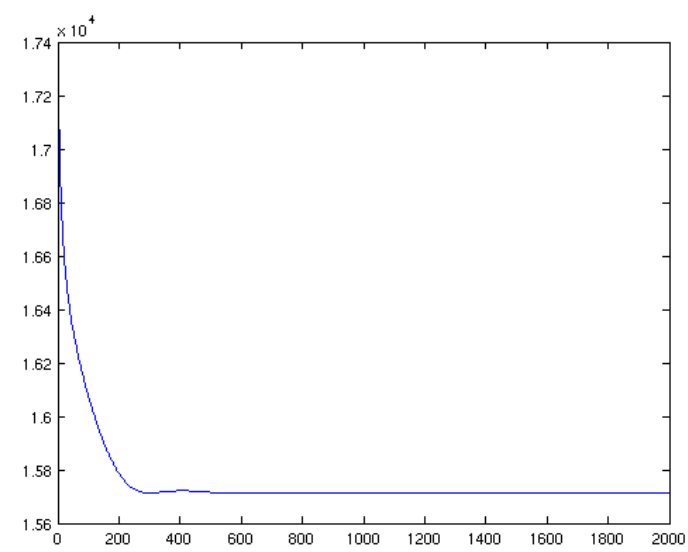

Figure 10. Convergence of the algorithm. On the $y$-axis the value of $J\left(u_{k}\right)$. On the $x$-axis the number of iterations 
Acknowledgments: The authors thank "Institut Pasteur de Paris" for providing them with biological images. The corresponding author warmly thanks Mikael Carlavan, Phd student of Ariana project at INRIA, to help him with the Matlab code.

\section{REFERENCES}

[1] G.Anzellotti. Pairings between measures and bounded functions and compensated compactness. Ann. Mat. Pura Appl. 135 (1983), 293-318.

[2] A.Braides. Г-convergence for beginners. Oxford University Press, New york (2000).

[3] A.Braides. Approximation of free discontinuity problems. Springer Lect. Notes in Math. Springer (1998) Vol1694.

[4] K.Bredies, K.Kunisch, T.Pock. Second order total generalized variation Siam Journal on Imaging Sciences, 3 2010, 492-526.

[5] G.Aubert, D.Graziani. Variational approximation for detecting point-like target problems. ESAIM: Control, Optimisation and Calculus of Variations, 17 2011, pp 909-930.

[6] M.Carriero, A.Leaci, F.Tomarelli A second order model in image segmentation: Blake \& Zisserman functional Variational Methods for Discontinuous Structures, Progress in Nonlinear Differential Equations and their Applications 25, Birkäuser, (1996), 57-72.

[7] G.Dal Maso. Introduction to Г-convergence. Birkhäuser, Boston(1993).

[8] G.Q.Chen, H.Frid Divergence-measure fields and conservation laws. Arch. Rational Mech. Anal. 147 (1999), 35-51.

[9] G.Q.Chen, H.Frid. On the theory of divergence-measure fields and its applications. Bol. Soc. Bras. Math. 32 (2001), 1-33.

[10] G.Dal Maso, F.Murat, L.Orsina, A.Prignet. Renormalized solutions of elliptic equations with general measure data. Ann. Scuola Norm. Sup. Pisa Cl. Sci, 28 (1999), 741-808.

[11] E.De Giorgi, T.Franzoni. Su un tipo di convergenza variazionale. Atti Accad. Naz. Lincei Rend. Cl. Sci. Mat. Natur. 58 (1975), 842-850.

[12] E.De Giorgi, T.Franzoni. Su un tipo di convergenza variazionale. Rend. Sem. Mat. Brescia 3 (1979), 63-101.

[13] L.C.Evans, R.F. Gariepy. Measure Theory and Fine Properties of Functions. CRC Press (1992).

[14] D.Graziani, Existence result for a free-discontinuity energy governing the detection of spots in image processing Applicable Analysis: An International Journal, (2012) DOI:10.1080/00036811.2011.653794

[15] D.Graziani, L.Blanc-Féraud, G.Aubert. A formal $\Gamma$-convergence approach for the detection of points in 2-D images. Siam Journal of Imaging Science 3 (2010), pp. 578-594.

[16] C.Goffmann, J.Serrin. Sublinear functions of measures and variation integrals. Duke Math J. 31 (1964), 159-178.

[17] J.Heinonen, T.Kilpeläinen, O.Martio. Nonlinear Potential Theory of Degenerate Elliptic Equations. Oxford University Press, (1993).

[18] Y.Nesterov. A method for unconstrained convex minimization problem with the rate of convergence $O\left(\frac{1}{\kappa^{2}}\right)$. Doklady AN SSSR 547 (1983), 543-547.

[19] Y. Nesterov. Smooth minimization of non-smooth functions. Mathematic Programming, Ser. A, 103 (2005) ,127-152.

[20] G.Stampacchia Le problème de Dirichlet pour les equations elliptiques du second ordre à coefficients discontinuous. Ann. Inst. Fourier (Grenoble), 15 (1965), 180-258.

[21] P.Weiss. Algorithmes rapides d'optimisation convexe. Application à la restauration d'images et à la détection de changements. Phd Thesis (2008).

[22] P. Weiss, L. Blanc-Féraud, G. Aubert. Efficient schemes for total variation minimization under constraints in image processing. SIAM journal on Scientific Computing 31 (2009), 2047-2080.

[23] W.Ziemer. Weakly Differentiable Functions. Springer-Verlag, New york (1989). 